\title{
Cardiotherapeutical results from the coupled BIOMED and GUYTON models
}

\author{
J. Werner, C. Welp, D. Böhringer \& M. Hexamer \\ Institute of Biomedical Engineering, Medical Faculty, \\ Ruhr-University Bochum, Germany
}

\begin{abstract}
The GUYTON model is still the most comprehensive cardiovascular model with a solid physiological background. However, it does not account for short term pulsatile processes. We therefore developed a pulsatile model (BIOMED) of the cardiovascular system and coupled it to the well documented modules of the GUYTON model. The BIOMED model focuses on the systolic wall tension progression, subject to the Frank-Starling- and Hill-mechanisms, and on the simulation of the processes due to the cardiac valves.

After successful validation of the dynamics and the steady-state behaviour of the decisive variables, pressure, volume and flow, by comparing them with published experimental and clinical recordings, we used the coupled systems to gain insight into the influences of impaired valve function and ventricular relaxation disorders on cardiac output, the importance of atrial contraction to ventricular filling, the significance of the choice of the adequate cardiac pacemaker and the need for an implementation of an av-delay, dependent on cardiovascular strain, in pacemakers.

This contribution focuses on the problem that pacemaker patients suffering from diastolic relaxation disorders are particularly dependent on an adjusted avdelay setting. The simulation results suggest the use of the derivative of ventricular pressure, correlating with peak endocardial acceleration, to control av-delay adaptation.

Keywords: pulsatile model, Guyton model, pacemaker, av-delay, relaxation disorder, peak endocardial acceleration.
\end{abstract}




\section{The GUYTON model}

The Guyton model (cf. Fig. 1, left part) is probably the most comprehensive model of the human cardiocirculatory system based on solid physiological knowledge. It was first published in 1972 [1] and was modified by the author [2, 3 ] and by users of the model. It excels by taking into account the interaction of the cardiocirculatory system with the autonomic and endocrine system, with metabolism, respiratory and kidney functions. The Guyton model consists of 17 modules containing about 600 physiological parameters and variables. It was originally written in FORTRAN, but was later translated into " $\mathrm{C}$ ” by Guyton and his colleagues. The kernel of the model is the "circulatory dynamics" module. The module "autonomics" refers to control of cardiocirculation via arterial baroreceptors, peripheral and central chemoreceptors and ergoreceptors (physical exercise). "Volume receptors" take into account the effect of the right atrial volume on the venous tone and the arterial resistance. The block "metabolism" summarizes oxygen delivery to muscle and non-muscle tissue, and local blood flow control (short-term via hypoxia-mediated vasodilatation, mid-term via tissue mediators, long-term via structured tissue changes). The "lung"-module mainly simulates the oxygen binding process in the erythrocytes. Further modules comprise hormonal, renal and electrophysiological processes.

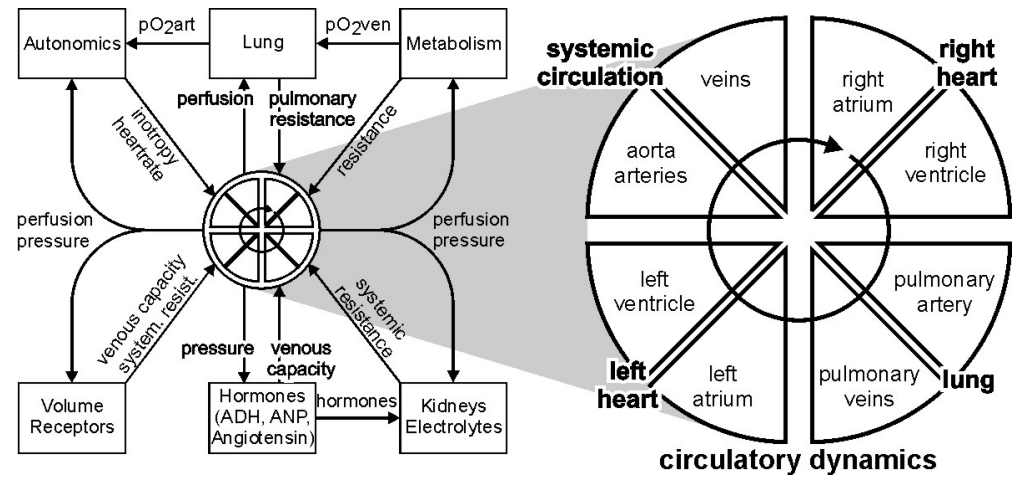

Figure 1: $\quad$ The coupled GUYTON and BIOMED model.

Unfortunately the GUYTON model does not consider the beat-to-beat processes crucial to the analysis of regulatory effects within the heart. Therefore we decided to develop the pulsatile BIOMED model (cf. Fig. 1, right part) which could functionally substitute the non-pulsatile "circulatory dynamics" component of the GUYTON model. The motivation for this was the need to develop predictive criteria for the short- and long-term effects of cardiotherapeutical treatments. After general validation and tests of plausibility of physiological and pathophysiological phenomena, we first used the coupled system to compute and to predict various effects of electrotherapy, such as the importance of atrial stimulation (versus or in combination with ventricular stimulation) and of an 
adequate atrioventricular delay, and to characterize and optimize sensorcontrolled pacing.

\section{The BIOMED model}

In the BIOMED model all vessels carrying oxygenated blood from the left part of the heart (left ventricle) into the systemic circulation (aorta, arteries, arterioles, capillaries) are merged and considered as "aorta compartment" (Fig. 1, right part). Similarly, blood vessels in which deoxygenated blood reverts to the heart (venules, veins) are combined in the "veins compartment". Since the process of respiration is not explicitly included in the pulsatile model, the vessel structures are scaled down to the units pulmonary artery and pulmonary veins which are two separate compartments, respectively.

The anatomy of the heart leads to the formation of four cardiac segments, the left atrium, the left ventricle, the right atrium and the right ventricle. Altogether the circulatory model consists of eight linked compartments with the basic variables pressure, flow and volume being a function of time within the heart cycle.

As the systemic blood circulation is a function of the cardiac stroke volume, the modelling of the heart kinematics, particularly the time flow of the heart contraction as well as the associated changes in the heart chamber's geometry has to be taken into account. Although this model uses a simplified heart chamber geometry in terms of a hollow sphere, bringing about a $25 \%$ lower wall tension [4] comparing it to a more realistic shape of a spheroid [5] for equal pressures and volumes, the approach is justifiable since the wall tension is not a resulting variable of the simulation itself, but is the driving input parameter to the model. The inner radius $r$ [cm] changes with systole and diastole as a result of the changes in atrial / ventricular volume $V_{\text {comp }}[\mathrm{ml}]$. Since the volume of each heart chamber's wall $V_{\text {wall }}[\mathrm{ml}]$ is considered to be constant, its wall thickness $d$ [cm] has to decrease during diastole and increase during systole, respectively. The following equations (1) and (2) describe this context:

$$
\begin{aligned}
& r(t)=\left(\frac{3}{4 \pi} V_{\text {comp }}\right)^{\frac{1}{3}} \\
& d(t)=\left(\frac{3}{4 \pi} V_{\text {wall }}+r^{3}\right)^{\frac{1}{3}}-r .
\end{aligned}
$$

The pressure inside the cardiac cavities consists of an active and passive pressure component. Defining the blood flow proportions into and out of the compartment as $\phi_{\text {in }}[\mathrm{ml} / \mathrm{min}]$ and $\phi_{\text {out }}[\mathrm{ml} / \mathrm{min}]$, respectively, the continuity equation can be written as follows:

$$
V_{\text {comp }}=\int \frac{d V_{\text {comp }}}{d t}=\int\left(\phi_{\text {in }}-\phi_{\text {out }}\right) d t
$$


Depending on the changes in volume the passive pressure component is obtained using the pressure-volume relationships [6, 7] for each of the four heart segments. The dynamic characteristics of the heart cycle with systole and diastole result from the active pressure component $p_{\text {active }}(t)[\mathrm{mmHg}]$ which is derived from an appropriate wall tension $\sigma(t)[\mathrm{mmHg}]$ (Fig. 2) for each motional pattern of the heart and Laplace's law

$$
p_{\text {active }}(t)=\sigma(t) \cdot \frac{2 d r+d^{2}}{r^{2}} .
$$

According to the wall movement during systole and diastole the value of the primary wall tension $\sigma_{\text {prim }}(t)$ is defined by Eq. (5) in which strengthfac [mmHg] is the maximum force that the heart muscle is able to generate under optimal preload with no afterload present, the nondimensional parameter for the relative autonomic activity symp having a value of 1 for the resting condition.

$$
\begin{aligned}
& \sigma_{\text {prim }}(\text { diastole })=0 \\
& \sigma_{\text {prim }}(\text { systole })=\text { strengthfac } \cdot \text { symp }
\end{aligned}
$$

To simulate the contraction and relaxation dynamics, time constants for the rise and decay of this rectangular function are implemented leading to a first order system, the potential wall tension $\sigma_{\text {pot }}(t)[\mathrm{mmHg}]$.

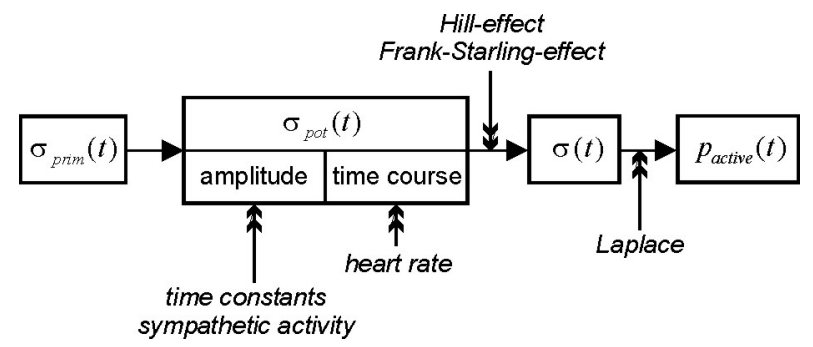

Figure 2: $\quad$ Formation of the active pressure component pactive( $\mathrm{t}$ ).

The Hill effect describes the correlation between the cardiac muscle shortening velocity, calculated in the model from the first derivative of the atrial / ventricular circumference, and the cardiac muscular strength. Since this factor depends on the relative autonomic activity, the characteristic Hill curve is adapted to the actual physical strain by alterable slopes. The greater the strain (symp $>1)$, the greater the muscle shortening velocity and therefore the greater the muscular strength is. In addition the adaptation of the heart to acute preload changes, called the Frank-Starling mechanism, is implemented in the model using a scaling factor between zero and one, directly proportional to the heart chamber's inner radius. The Hill and the Frank-Starling factor are multiplied by the potential wall tension $\sigma_{p o t}(\mathrm{t})$ to yield the actual wall tension $\sigma(t)$ and thereby with Eq. (4) the active pressure component. As shown in Fig. 1 the pressure of 
each compartment is fed back to its upstream compartment to produce a pressure gradient $\Delta p$ which is the circulatory driving force.

Using the Navier-Stokes law for incompressible fluids the laminar blood flow $\phi(t)[\mathrm{ml} / \mathrm{min}]$ can be calculated from

$$
L \frac{d \phi}{d t}=\Delta p-R \phi-\frac{\rho}{2}\left(v_{0}^{2}-v_{1}^{2}\right)
$$

with the pressure gradient $\Delta p[\mathrm{mmHg}]$, the resistance to flow $R\left[\mathrm{mmHg}^{*} \mathrm{~min} / \mathrm{l}\right]$, the inertance $L\left[\mathrm{mmHg}^{*} \min ^{2} / \mathrm{l}\right]$, and the density $\rho[\mathrm{kg} / \mathrm{l}]$. The active pressure gradient $\Delta p$, which is reduced by a pressure drop due to viscosity induced friction $(R \phi)$ and a Bernoulli-fraction $\left(\rho / 2\left(v^{2}{ }^{-} v^{2}\right)\right)$, accelerates the blood column.

In most pulsatile models of the human circulatory system $[5,8,9]$ the difference in blood flow velocity is replaced by the quotient of blood flow $\phi$ $[1 / \mathrm{min}]$ and the cross sectional area $A\left[\mathrm{~cm}^{2}\right]$ so that Eq. (6) can be simplified to

$$
L \frac{d \phi}{d t}=\Delta p-R \phi-\frac{\rho}{2}\left(\frac{\phi}{A}\right)^{2} .
$$

Eq. (7) is used for the compartments right ventricle, pulmonary artery, left ventricle and aorta. In the case of the aorta, the impedance was included modelling three successive compartments with decreasing compliances.

Within the left / right atrium, Eq. (7) can be further simplified, since the contribution of the blood acceleration is negligible $(<1 \mathrm{mmHg})$, leading to

$$
L \frac{d \phi}{d t}=\Delta p-R \phi .
$$

In all other compartments

$$
\Delta p=R \phi
$$

was applied.

As mentioned earlier, the volume-pressure relationships were used to obtain the compartments' active pressures with two exceptions, the pulmonary vein and the veins. Their pressures were derived from

$$
p(t)=\frac{V_{\text {comp }}-V_{0}}{c},
$$

in which $V_{\text {comp }}[\mathrm{ml}]$ is the compartment's volume, $V_{0}[\mathrm{ml}]$ the unstressed volume and $c[\mathrm{ml} / \mathrm{mmHg}]$ the compliance.

The functioning of the heart valves was explicitly modelled by allowing negative flow values in combination with previously defined dead space volumes for each valve. At the beginning of systole, the blood flow through the valve is integrated to add up to its dead space volume. Once this volume is completely filled, the valve is considered to be fully opened and the incoming blood flow 
raises the compartment's volume. During early diastole the negative pressure gradient between the considered compartments causes a reflux so that the valve is gradually shut according to its declining dead space volume. Valvular closure is only complete when the dead space volume is zero. This approach offers the possibility to simulate functional valve insufficiencies by increasing the valve's dead space volume.

\section{Evaluation}

The pulsatile model was developed to simulate physiological variables such as pressure, flow and volume under different conditions (physical strain or pathophysiological phenomena). These variables must first be evaluated with regard to their steady state values and their dynamic behaviour under resting conditions. Blood flow velocities are well documented in literature [e.g. 11, 12] for various locations in the cardiovascular system, since they can be measured noninvasively using Doppler ultrasound. In order to compare the simulated blood flows $\phi_{\text {sim }}[1 / \mathrm{min}]$ to these values, they need to be converted into mean blood flow velocities $v_{\text {mean }}[\mathrm{cm} / \mathrm{s}]$ with the simplified approach of a constant functional area $A_{\text {func }}\left[\mathrm{cm}^{2}\right]$ representing the cross sectional area of complex anatomical locations, e.g. the aortic cross sectional area, with

$$
v_{\text {mean }}=\frac{\phi_{\text {sim }}}{A_{\text {func }}} \text {. }
$$

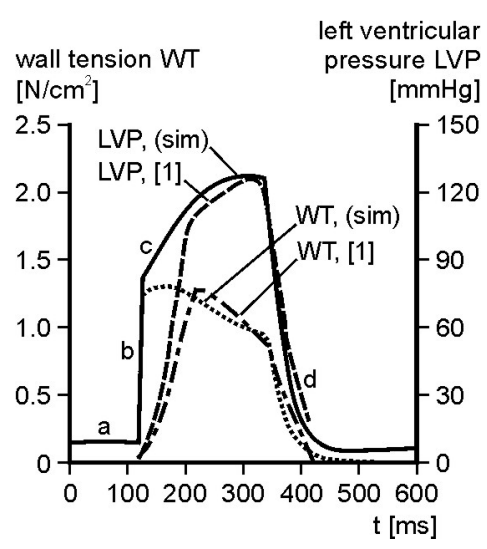

Figure 3: Left ventricular pressure LVP and corresponding wall tension WT: Comparison of the simulated dynamics with measured values from [1].

It turned out that the steady state values of the simulation results are within the $95 \%$ confidence interval of the "healthy" population. In a next step the dynamics of the pressure development during a heart cycle of all heart chambers were evaluated. In Fig. 3 the left ventricular pressure (LVP) and the corresponding 
wall tension (WT) are demonstrated. Evidently, the approach of defining wall tensions for each heart chamber depending on characteristic points in the heart cycle and above all on the Hill / Frank-Starling effects is able to produce physiological pressure curves and the characteristic "shoulder" in the wall tension's course.

Starting with the process of atrial filling of the left ventricle (a) in which the LVP is slightly increased, the isovolumetric contraction phase (b) follows with a steep pressure increase. At the same time the wall tension reaches its maximum value of about $1.25 \mathrm{~N} / \mathrm{cm}^{2}$. During the ejection phase (c) the LVP still rises until it reaches a maximum value of about $125 \mathrm{mmHg}$, marking the start of the ventricular diastole $(\mathrm{d})$ characterized by a steep pressure decay. Since the radius of the left ventricular chamber decreases and the wall thickness increases during the ejection phase, the wall tension decreases continuously according to Eq. (4).

There are various cardiac pathophysiological phenomena whose impact on the atrial / ventricular filling process or the cardiac stroke volume can be investigated with the aid of this model. It has been reported [13] that a cardiac insufficiency decreases the importance of the atrial systole on the ventricular filling process. Fig. 4 confirms this finding and illustrates the effect of a right heart failure on the pressure gradient between the veins close to the heart and the right atrium $\left(\Delta p_{R A}\right)$. The early diastolic pressure gradient in the right atrium is termed $\mathrm{E}$ and the pressure gradient resulting from the atrial contraction is termed A. The right heart failure was simulated by reducing the maximum systolic wall tension of the right ventricle by $40 \%$ resulting in a reduced stroke volume of the right heart and thereby an afflux of blood. This surplus of blood causes raised filling pressures for the right atrium / ventricle during diastole (Fig. 4: E is shifted towards E') so that in case of right heart failure the early diastolic influx into the atrium / ventricle accounts for nearly their entire filling volume and the atrial systolic pressure hardly exceeds the prevalent diastolic ventricular pressure (see "A" in Fig. 4).

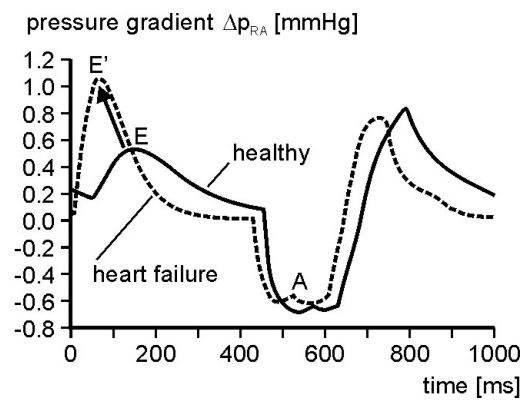

Figure 4: Simulated pressure gradient $\Delta \mathrm{pRA}$ between the veins and the right atrium in healthy/right heart failure subjects $\left(E, E^{\prime}=\right.$ early diastolic pressure gradient, $\mathrm{A}=$ atrial systolic pressure gradient). 
The pulsatile model as such is an open loop model, as no circulatory regulation mechanisms are implemented. However, by coupling the pulsatile model with the GUYTON model, phenomena which depend on the circulatory regulation can be investigated.

\section{Cardiotherapeutical application}

After successful validation of the dynamics and the steady-state behaviour of the decisive variables, pressure, volume and flow, we used the coupled systems to gain insight into the influences of impaired valve function and ventricular relaxation disorders on cardiac output, the importance of atrial contraction to ventricular filling, the significance of the choice of the adequate cardiac pacemaker $[14,15]$, and the need for an implementation of an av-delay, dependent on cardiovascular strain, in pacemakers. In this contribution the focus is laid on the problem that pacemaker patients suffering from diastolic relaxation disorders are particularly dependent on an adjusted av-delay setting. The simulation results suggest the use of the derivative of ventricular pressure, correlating with peak endocardial acceleration, to control av-delay adaptation.

The human cardiovascular system consists of multiple closed loops enabling the body to adapt the cardiac output to the prevalent physical strain by varying the heart rate or increasing / decreasing the cardiac contractility. Manifold cardiac disorders exist (e.g. av block) which are treated with pacemaker applications beside diverse drug therapies. Patients suffering from sinus node incompetence or an av block (III) depend on pacemakers which trigger the ventricular systole according to sensed physical parameters (e.g. acceleration). Since many of these patients (e.g. elderly people [2]) also suffer from diseases accompanied by diastolic relaxation disorders, their sensitivity to the pacemakers' av-delay settings was investigated in particular.

The relaxation disorders were simulated by extending the time constant (TC) for the end diastolic relaxation of the left ventricular wall tension. Fig. 5A shows the cardiac output $C O$ [1/min] subject to av-delay [ms] for three different stages of relaxation disorders: no relaxation disorder $(\mathrm{TC}=18 \mathrm{~ms})$, slight relaxation disorder $(\mathrm{TC}=54 \mathrm{~ms}$ ) and serious relaxation disorder $(\mathrm{TC}=90 \mathrm{~ms})$. Apparently persons with no relaxation disorders are less sensitive to av-delay settings, since the cardiac output remains on a high level of about $5 \mathrm{l} / \mathrm{min}$ throughout the range of av-value settings above $100 \mathrm{~ms}$. However, paced hearts with impaired myocardial relaxation would benefit from an exercise dependent av-delay because their cardiac output significantly decreases with increasing av-delays.

One promising physiological parameter for an av-delay adaptation approach is the derivative of the change in right ventricular pressure $d p_{R V} / d t[\mathrm{mmHg} / \mathrm{s}]$ [16], which correlates with the so called PEA (peak endocardial acceleration). It is well known that the PEA, being a function of the av-delay, has a steep decay in the optimal av value range in terms of the hemodynamics. The coupled system also predicts this effect for $d p_{R V} / d t$ (see Fig. 5B, grayish squares), since its first steep decay occurs in av-delay ranges which yield the highest cardiac outputs for 
patients with a slight diastolic relaxation disorder (cf. Fig. 5A). Furthermore this range is dependent on the degree of physical strain.

A cardiac output $\mathrm{CO}[1 / \mathrm{min}]$

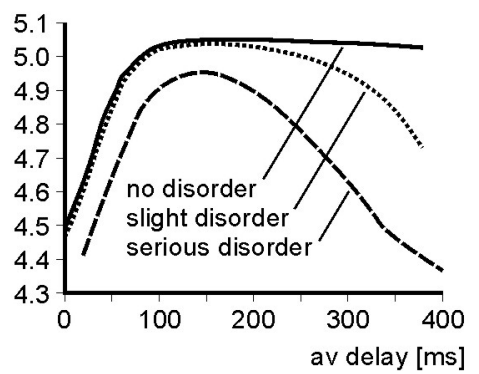

$\mathrm{B} \mathrm{dp}_{\mathrm{Rv}} / \mathrm{dt}[\mathrm{mmHg} / \mathrm{s}]$

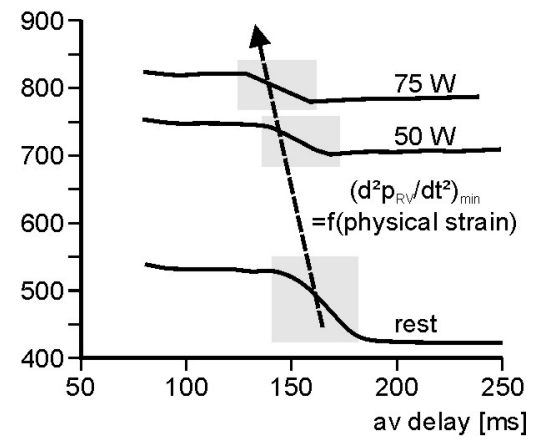

Figure 5: Simulation of ventricular relaxation disorder. A: Simulated cardiac output CO subject to av-delay for three different stages of ventricular relaxation disorders: no disorder (control), slight disorder, serious disorder. B: Simulated pressure gradient $\mathrm{dpRV} / \mathrm{dt}$ in the right ventricle for patients with a slight diastolic relaxation disorder under different physical strains: rest, $50 \mathrm{~W}$ and $75 \mathrm{~W}$ (Grayish squares $=$ optimal av-delay range).

\section{Conclusions}

Coupling the developed pulsatile BIOMED model to the established GUYTON model set the stage for diverse investigations on pathophysiological processes and cardiac electrotherapy. The application results outlined here showed that the adequate av-delay is of crucial importance in pacemaker technology, especially in patients suffering from ventricular relaxation disorders. While the coupled model is already an applicable tool for educational purposes, the simulation has to be further developed and tested before using it routinely for cardiotherapeutical considerations.

\section{Acknowledgments}

The project was supported by the DFG (German research community), grants No. WE 919/16-1 and HE 2713/4-1.

\section{References}

[1] Guyton, A.C., Coleman, T.G. \& Granger, H.J, Circulation: Overall Regulation. Ann. Rev. Physiol. 34, pp. 13-43, 1972. 
[2] Guyton, A.C., Annotation of Large Circulatory Model, (to be obtained by the author) 1993.

[3] Guyton, A.C. \& Taylor, C.E., Dynamics and Control of the Body Fluids, Saunders: Philadelphia, 1975.

[4] Huisman, R.M., Sipkema, P. \& Westerhof, N, Comparison of models used to calculate left ventricular wall force. Med. Biol. Eng. Comp.18, pp. 133-144, 1980.

[5] Guyton, A.C. \& Jones, C.E., Cardiac Output and its Regulation, Saunders: Philadelphia, 1973.

[6] Guyton, A.C., Textbook of Medical Physiology, Saunders: Philadelphia, 1991.

[7] Karsch, K.R. \& Scheufler, S., The right ventricle at rest and during exercise. Z. Kardiol. 7, pp. 485-490, 1979.

[8] Leaning, M.S., Pullen, H.E. \& Finkelstein, L., Modelling a complex biological system: the human cardiovascular system -1 . Methodology and model description. Trans. Inst. Meas. Contr. 5, pp. 72-83, 1983.

[9] Urbaszek, A. \& Schaldach, M., A numerical heart and circulation model to simulate hemodynamics for rate responsive pacing. Advances in Fluid Mechanics, Vol. 3, ed. H. Power, Computational Mechanics Publications: Southampton, Boston, pp. 129-159, 1995.

[10] Ursino, M., Interaction between carotid baroregulation and the pulsating heart: A mathematical model. Amer. J. Physiol. 275, pp. H1733-H1747, 1998.

[11] Järvinen, V.M. \& Kupari, M.M., Right atrial MR imaging studies of cadaveric atrial casts and comparison with right and left atrial volumes and function in healthy subjects. Radiology 19, pp. 137-142, 1994.

[12] Lentner, C., Heart and Circulation: Geigy Scientific Tables, Ciba-Geigy: Basel, 1992.

[13] Greenberg, B., Chatterjee, L., Parmley, W., Werner, J. \& Holly A., The influence of left ventricular filling pressure on atrial contribution to cardiac output. Am. Heart J. 98, pp. 742-751, 1979.

[14] Werner, J., Hexamer, M., Meine, M. \& Lemke, B., Restoration of cardiocirculatory regulation by rate-adaptive pacemaker systems. IEEE Trans. Biomed. Eng. 46, pp. 1057-1064, 1999.

[15] Werner, J., Böhringer, D. \& Hexamer, M., Simulation and prediction of cardiotherapeutical phenomena from a pulsatile model coupled to the Guyton circulatory model. IEEE Trans. Biomed. Eng. 49, pp. 430-439, 2002 .

[16] Menozzi, C., Tomasi, C., Brignole, M., Lolli, G. \& Bottoni, N, Cardiac contractility: concepts and advances in implantable system application. Proc. of the Vth southern symposium on cardiac pacing, ed. L. Pozzi: Rom, pp. 383-396, 1996. 